\title{
Intelligent Integration between Human Simulated Intelligence and Expert Control Technology for the Combustion Process of Gas Heating Furnace
}

\author{
Yucheng Liu ${ }^{1}$ and Yubin Liu $^{2}$ \\ ${ }^{1}$ College of Electrical \& Information Engineering, Chongqing University of Science \& Technology, Shapingba, \\ Chongqing 401331, China \\ ${ }^{2}$ School of Continuing Education, Panzhihua University, Panzhihua, Sichuan 617000, China \\ Correspondence should be addressed to Yucheng Liu; lych68782763@163.com
}

Received 6 December 2013; Accepted 14 January 2014; Published 27 February 2014

Academic Editor: Zhengguang Wu

Copyright (C) 2014 Y. Liu and Y. Liu. This is an open access article distributed under the Creative Commons Attribution License, which permits unrestricted use, distribution, and reproduction in any medium, provided the original work is properly cited.

\begin{abstract}
Due to being poor in control quality of the combustion process of gas heating furnace, this paper explored a sort of strong robust control algorithm in order to improve the control quality of the combustion process of gas heating furnace. The paper analyzed the control puzzle in the complex combustion process of gas heating furnace, summarized the cybernetics characteristic of the complex combustion process, researched into control strategy of the uncertainty complex control process, discussed the control model of the complex process, presented a sort of intelligent integration between human-simulated intelligence and expert control technology, and constructed the control algorithm for the combustion process controlling of gas heating furnace. The simulation results showed that the control algorithm proposed in the paper is not only better in dynamic and steady quality of the combustion process, but also obvious in energy saving effect, feasible, and effective in control strategy.
\end{abstract}

\section{Introduction}

The industrial furnace is a kind of device to be used to convert fuel into heat energy equipment. The combustion efficiency of fuel in the combustion process of the industrial furnace is directly related to the energy saving, emission reduction, and the natural ecological environment protection. That fuel burns in the mixing with air according to the best proportion way can achieve the control effect of energy saving and emission reduction. The energy loss is inevitable in the process of combustion. The oxygen of only 21 percent in the air contributes to the combustion; the other $79 \%$ nitrogen which does not participate in the combustion is also heated and discharged from the flue into the atmosphere. Therefore, it is necessary to minimize the input of air to avoid the energy consumption caused by the excess air so as to make the energy consumption reduced to the minimum degree. In general, the oxygen $\left(\mathrm{O}_{2}\right)$ content of $0.25 \%$ should be increased in the air to ensure full combustion. The above analysis is correct in terms of theory. However, because of the influence of various uncertainty factors in the practical control engineering, the control of the combustion process in accordance with collocation methods of the optimum proportion of fuel and air is technically difficult in the implementation of the project. The following is the discussion to the relevant problem.

\section{Combustion Process Cybernetics Characteristics}

In order to facilitate the discussion, take the control of the combustion process of the steel rolling gas heating furnace as an example to research into the cybernetics characteristics of the combustion process. In general, the mixed gas is selected as the fuel in the steel rolling heating furnace (blast furnace, coke oven, and converter) of metallurgical enterprises. When the fuel burning value is low, the appropriate natural gas with high burning value can be added into the fuel. The influence of change in the working situation, such as 
the blast furnace, the coke oven, and the converter, measurement error of the burning value parameter, and the changes in the environment and operating condition are uncertain. It is under the condition of the statistical hypothesis that the burning value parameters of the mixed gas are obtained. Though these parameters follow the corresponding statistical law, they are random variables. Their exact values at every moment are not known. The values of the gas burning value parameters generally obtained by using the unknown but bounded (UBBE) hypothesis also are in the bounded range. Their exact values are still not known. Because of being artificially simplified, the model obtained in this case is only a nominal model. Therefore, the combustion process of the gas heating furnace belongs to the structured uncertainty process or parameter uncertainty process. Some parameters of the control process are uncertain. And the values of these parameters can only be in a given range. In addition, the combustion process of gas heating furnace for steel rolling is a large inertia and large time-delay process. Being closely related to the amount of materials in the heating furnace, the inertia time parameters and the time-delay parameters actually are uncertain, which is a challenge to the control of the combustion process. In view of the large inertia and large time-delay process being difficult in the control engineering, many scholars have studied it and have achieved the gratifying research results [1-4].

\section{Control Strategy Selection of Combustion Process}

From the cybernetics characteristic mentioned above, it can be seen that it is difficult to construct the mathematical model of the uncertainty complicated combustion process. Therefore, it is impossible to use the methods and techniques based on the classical control theory or modern control theory to obtain the expected control effect of the uncertainty complicated combustion process of the gas heating furnace $[5,6]$. Because of its control algorithm being based on the strict mathematic model, the conventional control strategy such as PID control strategy is not advisable [7]. At present fuzzy control technology has been lots of successful application example of case. But there are some puzzles such as the selection of membership function for complex combustion process with uncertainty and large time delay as well as large inertia. And it is difficult completely to reflect the influence on the control algorithm for professional knowledge, actual experience, operating skill, and adjustment technique of the operator. It would lead to being low in precision and poor in robustness, and therefore it is not advisable to adopt fuzzy control for the uncertainty complicated combustion process of the gas heating furnace. The expert system (ES) can deal with each kind of qualitative, quantitative, accurate, and fuzzy information; can fully summarize the knowledge, experience, and technique of the operator; and can bring them into the control program. However, there are some puzzles such as how to obtain and express knowledge in constructing a perfect knowledge base. So it is unwise completely to adopt expert system control in the combustion process of the gas

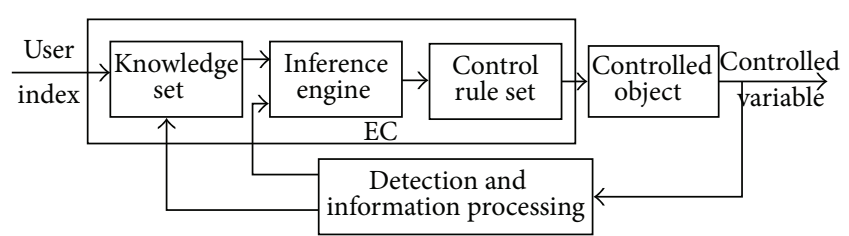

FIGURE 1: HSIC control model structure.

heating furnace, but its basic technique is meaningful to use for the reference. It is worth paying attention to human simulated intelligent controller (HSIC). It is effectively a sort of homomorphism transformation of the human body control system. In the actual operating process, the operator can timely make corresponding control action on the basis of the control effect and operating information of a previous moment. There is much information in a continual operating control process, which is useful to control. The operator can select some information having a characteristic meaning, namely, the characteristic information to make memory, so as to avoid large information storage space which is necessary to the conventional controller with the blind memory information. The experience shows that the characteristic memory has the implication relationship with control output, taking that it is available to eliminate the process error and make it not to produce the integral saturation phenomenon so as to reduce the lag caused by the integral. By means of HSIC, as long as the process change exists in deviation, then the characteristic model can make the perception for its change. It can find its corresponding control pattern through the recognition of the judgment. For the complicated combustion process of the gas heating furnace with many uncertainty factors, the strategy of HSIC should be considered as a better selection. As a result of characteristic information having reflected the dynamic characteristic of the whole combustion process, the corresponding control pattern can be found according to the characteristic reflected by characteristic model [8]. In terms of corresponding control patterns, the control algorithm that is suitable for the combustion process can be constructed, and thereby it can ensure the process to achieve the demand of control quality in dynamic and steady state. Aiming at the features of the combustion process of the gas heating furnace, based on the technical integration between human simulated intelligence and expert control technology, intelligence fusion control strategy was adopted in this paper [9-11] so as to ensure that the control of the combustion process is high in precision, short in transient process time, and strong in robustness as well as with no overshoot.

\section{Control Model Structure}

The generalized control model was selected as the control model of uncertainty complicated combustion process in the control strategy of intelligence fusion, and it was shown as in Figure 1. Being considered as an integrated control model $[12,13]$, the model made the fusion between knowledge model and mechanism. The superiority of control model lies in that 


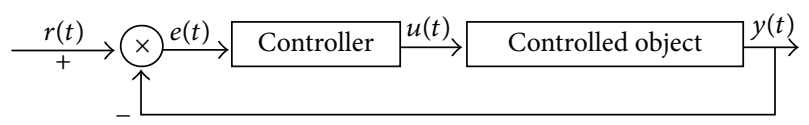

FIGURE 2: General control model structure.

it is unnecessary to know more prior knowledge for uncertain complicated combustion process of the gas heating furnace, and as long as it knows the process deviation and its change rate then it can immediately realize an effective control for the uncertainty complicated combustion process [14-16]. Figure 2 shows the structure of general control system, in which $r(t)$ is the process input, $y(t)$ is the process output, $e(t)$ is the process deviation, and $e(t)=r(t)-y(t)$. The process deviation and time change rate of the process deviation can construct the solving information space of control problem. In the control problem of HSIC, the controller of generalized control model is based on characteristic recognition of the solving information space such as, for instance, size of the process deviation, change direction of the process deviation, and change trend of the process deviation. If these features are known, then the corresponding control pattern can be selected, and therefore the corresponding control algorithm can be constructed so as to realize the high accurate and strong robust control for the uncertainty complicated combustion process of the gas heating furnace.

The generalized control model based on HSIC can fuse the technology of HSIC and expert system technique to make control system structure simplified. It can easily fuse the control rule and inference organization as well as knowledge base into an intelligent controller, and also it is convenient to adopt the production rule of "If < condition $>$ Then < action>" to establish knowledge set. Its outstanding advantages are that each piece of rule can independently be added, deleted, and revised, it is better in the modularity and naturality, there is no direct relation among the rules, and it owns strong adaptive nature for environment change. By means of basic characteristic combined open-loop and closed-loop control in HSIC, it can easily realize nimble multimodal control, and it strengthens the capability of judgment and inference. Therefore the strategy is suitable for the control feature of complex process with uncertainty.

\section{Basic Control Algorithm}

The control algorithm of control strategy based on the intelligence fusion can be summarized as two sorts of dynamic characteristic patterns. One Pattern of proportion and half derivation (PH-D control pattern) whose execution condition is that if $e \bullet \dot{e} \geq 0$ and $e+\dot{e} \neq 0$ then PH-D control pattern, another pattern is half open-loop (HO control pattern) whose execution condition is that if $e \cdot \dot{e} \leq 0$ or $e=\dot{e}=0$ then $\mathrm{HO}$ control pattern. The algorithm of prototype in the human simulated intelligent controller is the following.
The control rule set can be derived from the prototype of HSIC, and it can be expressed as follows:

$$
u= \begin{cases}K_{p} e+k K_{p} \sum_{i=1}^{n-1} e_{m, i} & (e \cdot \dot{e}>0 \cup e=0 \cap \dot{e} \neq 0) \\ k K_{p} \sum_{i=1}^{n} e_{m, i} & (e \cdot \dot{e}>0 \cup \dot{e}=0),\end{cases}
$$

where $u, K_{p}, k, e, \dot{e}$, and $e_{m, i}$, respectively, represent the control output, proportion coefficient, constraint coefficient, system deviation, change rate of system deviation, and the value of the $i$ th deviation peak. Generally speaking, the design of human simulated intelligent controller can be summarized as the following basic steps.

(1) Establish the Design Target Trace. According to the user demand of control performance index such as rise time, overshoot, and steady precision, it first determines the ideal response process of unit step. And after that, it transforms the ideal response process of unit step into the time phase space of $e-\dot{e}-t$ and constitutes the deviation time phase trace.

(2) Establish the Characteristic Model. According to the position of target trace in deviation phase plane of $e-\dot{e}$ or the position in deviation time plane of $e-t$, as well as different layers of the controller such as running control layer and task adaptation layer, the characteristic basic layer should be determined and the partition of the characteristic state layer also be performed so as to constitute a characteristic model of a different level.

(3) Design the Control Rule and Control Mode Set. Aiming at the difference between instantaneous index and certain characteristic state in the characteristic model located by moving state of the system, as well as moving trend of ideal trace, it simulates the decision behavior of human simulated control and designs the control mode. For the design of controller in running layer, the target trace of ideal deviation is shown by broken line in Figure 3. In order to make the actual error trajectory be consistent with the ideal target error trajectory as much as possible, it can take the following control countermeasures.

If the deviation is getting great, corresponding to area (1) in Figure 3, then the Bang-Bang control mode would be adopted; namely, control action should be as large as possible.

If the deviation and its change rate are getting very small, corresponding to area (5) in Figure 3, then the PI algorithm would be adopted so as to eliminate the system steady deviation.

If the deviation is getting larger, corresponding to area (2) in Figure 3, then proportional control mode would be adopted. And at the same time, it has to introduce a weak differential coefficient so as to endure the deviation change rate not being too large.

In the process of reducing deviation, if the deviation change rate is getting lower or equal to prespecified rate, corresponding to area (4) in Figure 3, then the proportional and differential control mode would be adopted. 


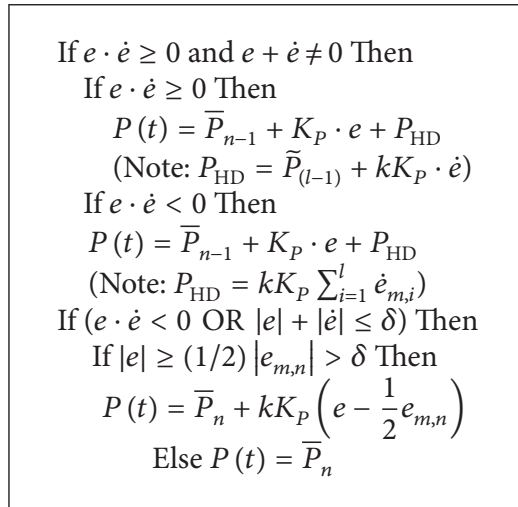

Algorithm 1

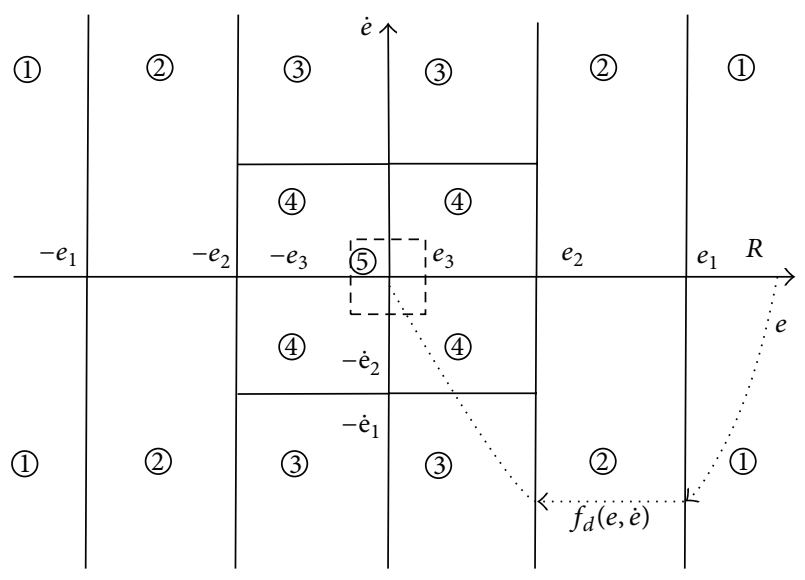

FIGURE 3: Running control characteristic model.

In the process of reducing deviation, if the deviation change rate is getting great than prespecified rate, corresponding to area (3) in Figure 3, then the strong differential control based on proportional mode has to be introduced so as to reduce deviation change rate as soon as possible.

Therefore the system running can be expressed as lots of control mode according to the deviation and deviation change rate of the complex process with uncertainty. Based on the selection of the above control strategy, the control thought is that a different control strategy should be adopted for the different characteristic pattern of system deviation. Summarizing hominine control experience based on prototype algorithm, the set of control rule in complex process with uncertainty is a suit of control rule corresponding to different characteristic status. Combining with the features of the combustion process of the gas heating furnace and introducing expert system technique into controller, after the expert knowledge, practice experience, operating skill, and adjusting technique of operators are fused in the algorithm, the detailed control algorithm can be formed easily by means of structured English description method. Its control algorithm can make corresponding decision according to the size, direction, and change trend of the system deviation.
It simulates some features and functions of a human being from two aspects of control structure and control behavior. And the control algorithm can select the control pattern and control execution algorithm of next control period according to the result of dynamic characteristic recognition. The advantages of the above algorithm are that it needs not more operator's prior knowledge of the uncertainty complicated combustion process of the gas heating furnace, and it is faster in process response, higher in control precision, and stronger in robustness. And the detailed control algorithm can be summarized by structured English description method as shown in Algorithm 1.

In this algorithm, $\bar{P}_{n}=k K_{P} \sum_{i=1}^{l} e_{m, i}$; the $P$ is output (to controlled process), $e$ is input (system deviation signal), $\dot{e}$ is first order differentiation with time, $e_{m, i}$ is the $i$ th extreme value, $K_{p}$ is the proportional gain, $k$ is restraint coefficient, $P_{\mathrm{HD}}$ is half-derivative amount in the pattern of P-HD, $\dot{e}_{m, i}$ is the $i$ th extreme value of $\dot{e}$ in the P-HD pattern, and $\widetilde{P}_{l}$ is the needed holding value of the $l$ th output component of half derivative in the pattern of P-HD. Define $\widetilde{P}_{0}=0$ (memory of derivative extreme value). $i, l$ and $n$ are the nature number. $\delta$ is the sensitivity bound of controller.

\section{Simulation Experiment}

The combustion process of the gas heating furnace is a complex uncertainty process with large pure lag and great inertia. In order not to lose generality, the control problem can transformed as examining the robustness of control algorithm. The control algorithm with strong robustness owns very strong adaptation capacity, and the influence caused by the change of the control parameter is so small as to hardly affect the control quality of the system. In order to simplify the analysis, the representative in the control engineering twoorder lag process model was selected as an example to study the robustness of the control strategy. Therefore, the model of controlled combustion process of the gas heating furnace can be assumed as follows:

$$
G(s)=\frac{K e^{-\tau s}}{\left(T_{1} s+1\right)\left(T_{2} s+1\right)},
$$




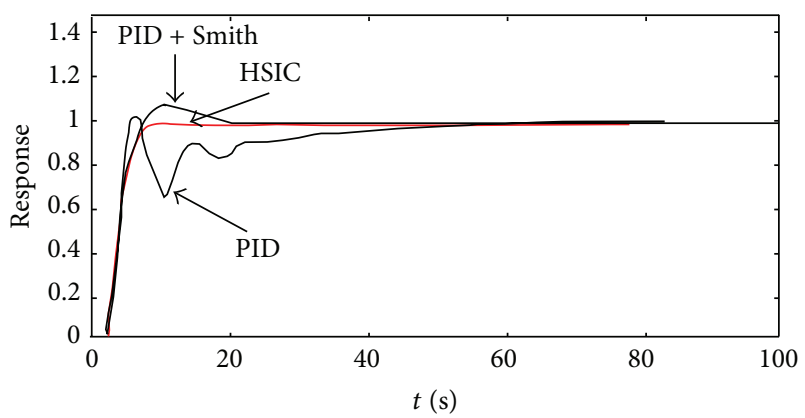

Figure 4: Process unit step response $(\tau=2 \mathrm{~s})$.

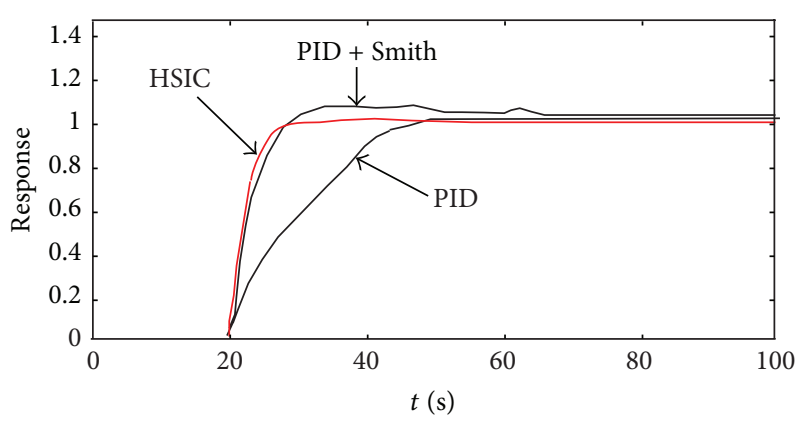

FIgURE 5: Process unit step response $(\tau=20 \mathrm{~s})$.

in which $K=4.134, T_{1}=1 \mathrm{~s}$, and $T_{2}=2 \mathrm{~s}$. In order to facilitate the comparison, the intelligence integration control algorithm (simplified as HSIC), conventional PID control strategy, and PID + Smith optimal control strategy are separately used to realize the control of the combustion process of the gas heating furnace. Then, through the comparison of the output response, the control strategy with strong robustness can be selected.

In the simulation experiment, respectively, take $\tau=2 \mathrm{~s}$ and $\tau=20 \mathrm{~s}$; the response curves of simulation experiment were, respectively, shown in Figures 4 and 5.

Figure 4 showed that the response curve of the PID control has a serious oscillation and its adjusting time is too long, so it cannot be used in the process control; the response curve of the PID + Smith optimal control has a serious overshoot; the response curve of the HSIC has fast response speed, short adjusting time, and small steady-state error. Therefore, the fusion control strategy based on human simulated intelligence is a good quality control strategy. Figure 5 shows that, after the lag time being increased by 10 times, the fusion control strategy based on human simulated intelligence can still obtain excellent control quality, namely, fast response speed, short adjusting time, small steady-state error, and strong robustness; at this time, PID has not been used to control the process because the response speed is too slow and the adjusting time too long; the PID + Smith optimal control has serious overshoot and too long adjusting time, so it is not desirable. What Figure 6 shows are the output responses which have $\tau=15 \mathrm{~s}$ and the external interference of the pulse amplitude being 0.5 and pulse width being $0.2 \mathrm{~s}$. Figure 6 shows that, because of having fast response speed,

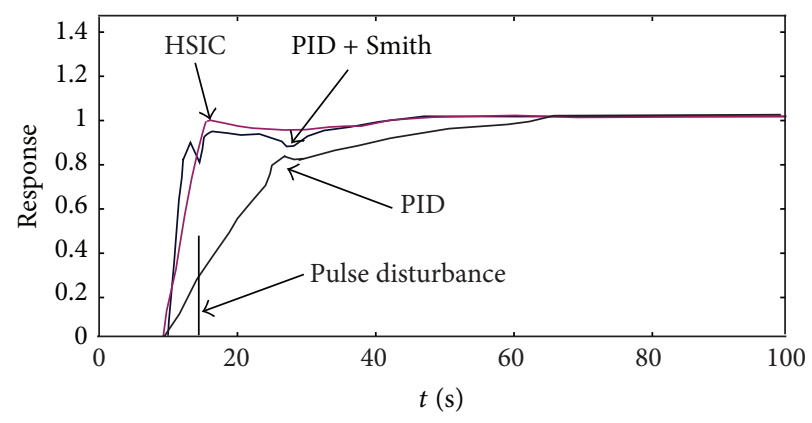

Figure 6: Process unit step response with the external disturbance $(\tau=15 \mathrm{~s})$.

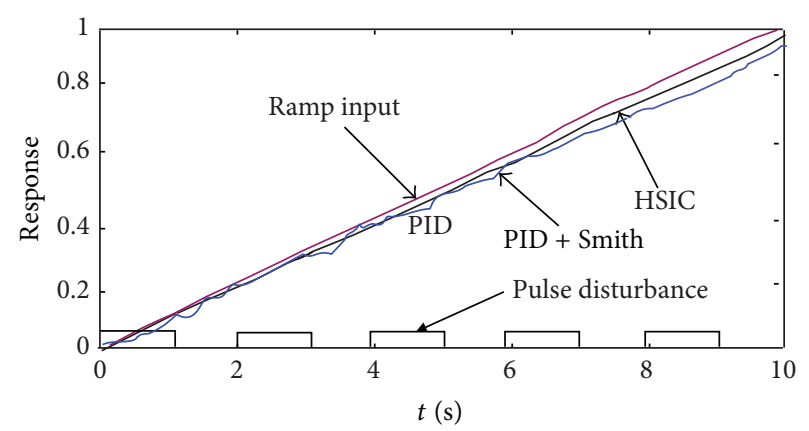

FIGURE 7: Process unit ramp response with external disturbance $(\tau=$ $2 \mathrm{~s})$.

short adjusting time, and small steady-state error, the fusion control strategy based on human simulated intelligent control still can obtain excellent control quality under the external heavy disturbance, while the PID and PID + Smith control strategy already could not be used to control the process. What Figure 7 shows are the output responses which have $\tau=2 \mathrm{~s}$ and the external disturbance of the pulse amplitude being 0.5 and pulse width being $10 \mathrm{~s}$. Figure 7 shows that fusion control strategy based on human simulated intelligent control has a good tracking performance of the ramp input; its tracking error is small and stable. Figure 7 also shows that although the PID + Smith control can also track the ramp input, its tracking error is relatively large and very unstable.

\section{Conclusions}

It is very difficult and complex to control uncertainty process. The main reason is that it is difficult to establish mathematical model. The extent of difficulty in the control is closely related to the user's quality requirements for process control. In general, if the strong robust control strategy is used, as long as the range of change of the uncertainty quantity can be known without mathematical model, the deterministic control law or strong robust control algorithm which can meet the user's control quality requirements can also be designed by using intelligent control based on knowledge model. The engineering practice on the combustion process control of the gas heating furnace shows that the fusion control algorithm based on intelligent integration between human 
simulated intelligence and expert control technology has strong robustness. The use of the fusion control algorithm can ensure the dynamic and static control quality requirements of the gas heating furnace combustion process. The simulation experiment shows that using the control algorithm proposed in the paper can ensure obtaining a better effect in dynamic and steady control quality of the combustion control process response, and it is high in control precision, and short in transient process response. Under the condition of the external interference, compared with conventional PID control algorithm and the PID + Smith control algorithm, the intelligence fusion control algorithm has very strong robustness. Therefore, it is a better control algorithm for the control of a complex process with uncertainty.

\section{Conflict of Interests}

The authors declare that there is no conflict of interests regarding the publication of this paper.

\section{Acknowledgment}

This work was supported by Program for Innovation Team Building at Institutions of Higher Education in Chongqing (KJTD 201324).

\section{References}

[1] B. Hong, C. H. Hu, and X. P. Jiang, "Iterative multi-step prediction model based on theory of evidence," Control Theory \& Applications, vol. 27, no. 12, pp. 1737-1742, 2010.

[2] G. Hu, Y. Q. Liu, and Y. Q. Li, "The cause of formation, the classification and the control strategy for uncertainty control systems," Industrial Engineering Journal, vol. 4, no. 1, pp. 49-52, 2001.

[3] Y. Yuan, "Uncertain model for knowledge-based system," Application Research of Computers, vol. 26, no. 9, pp. 3381-3383, 2009.

[4] F. Xu, M. Y. Liu, W. B. Li, and X. K. Lei, "Research on digital redesign of the robust controller for parametric uncertain systems," Systems Engineering and Electronics, vol. 35, no. 1, pp. 156-160, 2013.

[5] Y. Dai, S. Shi, and N. Zheng, "Class of robust control strategies for robot manipulators with uncertainties," Acta Automatica Sinica, vol. 25, no. 2, pp. 204-209, 1999.

[6] P. Carmona, J. L. Castro, and J. M. Zurita, "FRIwE: fuzzy rule identification with exceptions," IEEE Transactions on Fuzzy Systems, vol. 12, no. 1, pp. 140-151, 2004.

[7] C. N. Dai, M. Yao, Z. J. Xie, C. H. Chen, and J. G. Liu, "Parameter optimization for growth model of greenhouse crop using genetic algorithms," Applied Soft Computing Journal, vol. 9, no. 1, pp. 13-19, 2009.

[8] N. S. Bhuvaneswari, G. Uma, and T. R. Rangaswamy, "Adaptive and optimal control of a non-linear process using intelligent controllers," Applied Soft Computing Journal, vol. 9, no. 1, pp. 182-190, 2009.

[9] Z. P. Liu, Q. Yang, and W. J. Yan, "Intelligent modeling and predictive control of pre-grinding system," Advanced Materials Research, vol. 433-440, pp. 2120-2127, 2012.

[10] R. Alcalá, J. Alcalá-Fdez, F. Herrera, and J. Otero, "Genetic learning of accurate and compact fuzzy rule based systems based on the 2-tuples linguistic representation," International Journal of Approximate Reasoning, vol. 44, no. 1, pp. 45-64, 2007.

[11] J. González, I. Rojas, H. Pomares et al., "Improving the accuracy while preserving the interpretability of fuzzy function approximators by means of multi-objective evolutionary algorithms," International Journal of Approximate Reasoning, vol. 44, no. 1, pp. 32-44, 2007.

[12] H. Ishibuchi and Y. Nojima, "Analysis of interpretabilityaccuracy tradeoff of fuzzy systems by multiobjective fuzzy genetics-based machine learning," International Journal of Approximate Reasoning, vol. 44, no. 1, pp. 4-31, 2007.

[13] E. Van Broekhoven, V. Adriaenssens, and B. De Baets, "Interpretability-preserving genetic optimization of linguistic terms in fuzzy models for fuzzy ordered classification: an ecological case study," International Journal of Approximate Reasoning, vol. 44, no. 1, pp. 65-90, 2007.

[14] K. H. Quah and C. Quek, "FITSK: online local learning with generic fuzzy input Takagi-Sugeno-Kang fuzzy framework for nonlinear system estimation," IEEE Transactions on Systems, Man, and Cybernetics B, vol. 36, no. 1, pp. 166-178, 2006.

[15] S. M. Zhou and J. Q. Gan, "Constructing accurate and parsimonious fuzzy models with distinguishable fuzzy sets based on an entropy measure," Fuzzy Sets and Systems, vol. 157, no. 8, pp. 1057-1074, 2006

[16] J. Casillas, O. Cordón, M. J. Del Jesus, and F. Herrera, “Genetic tuning of fuzzy rule deep structures preserving interpretability and its interaction with fuzzy rule set reduction," IEEE Transactions on Fuzzy Systems, vol. 13, no. 1, pp. 13-29, 2005. 


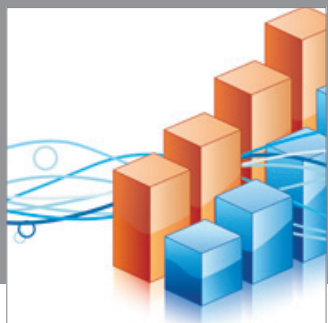

Advances in

Operations Research

mansans

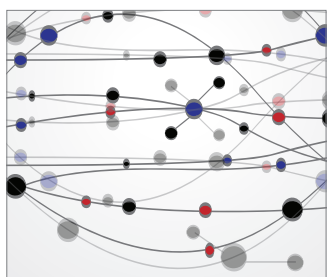

The Scientific World Journal
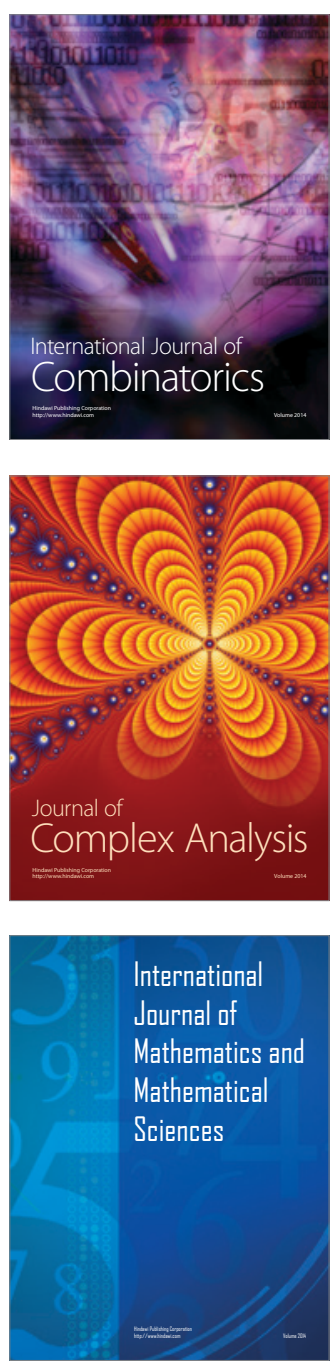
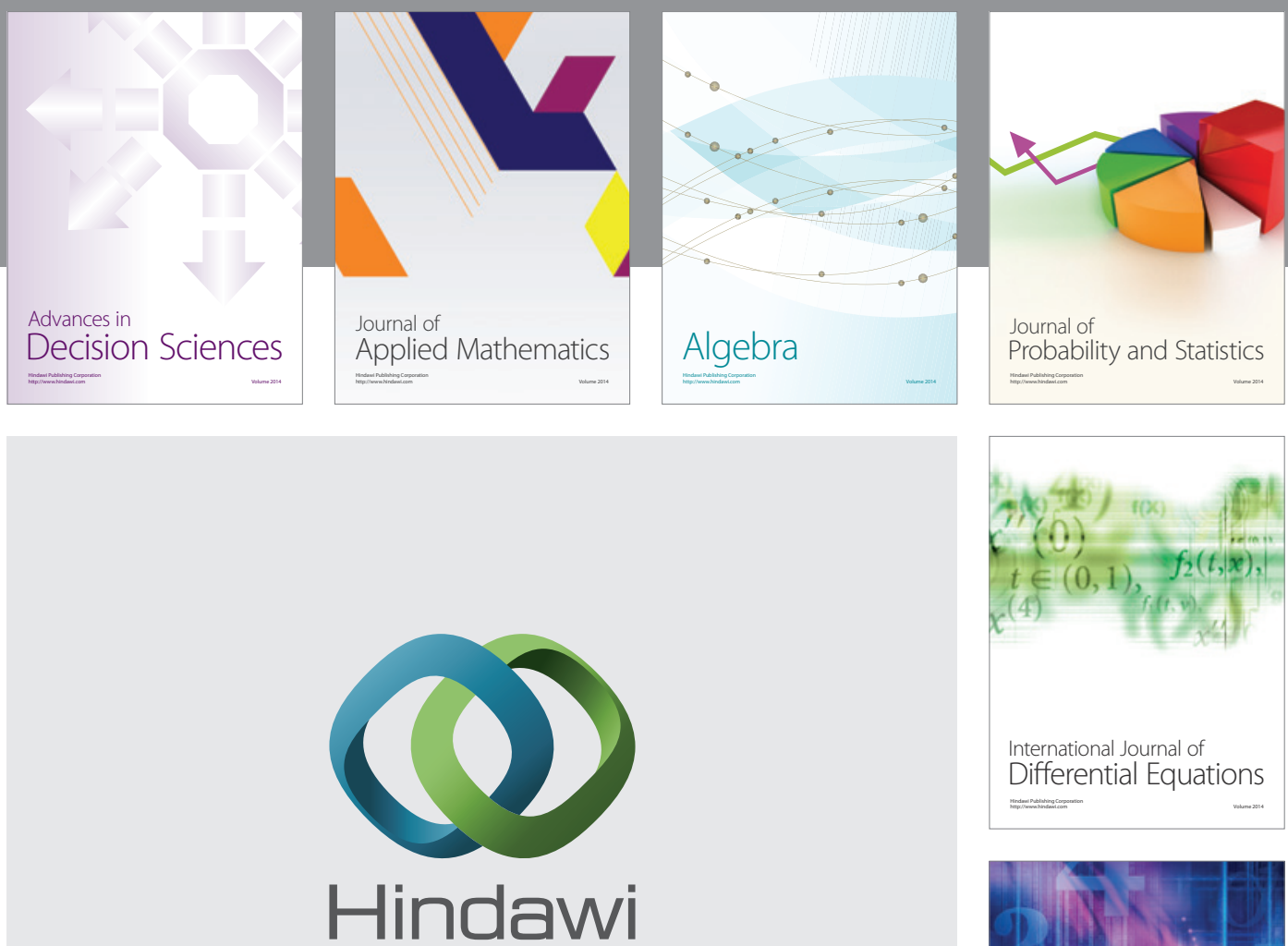

Submit your manuscripts at http://www.hindawi.com
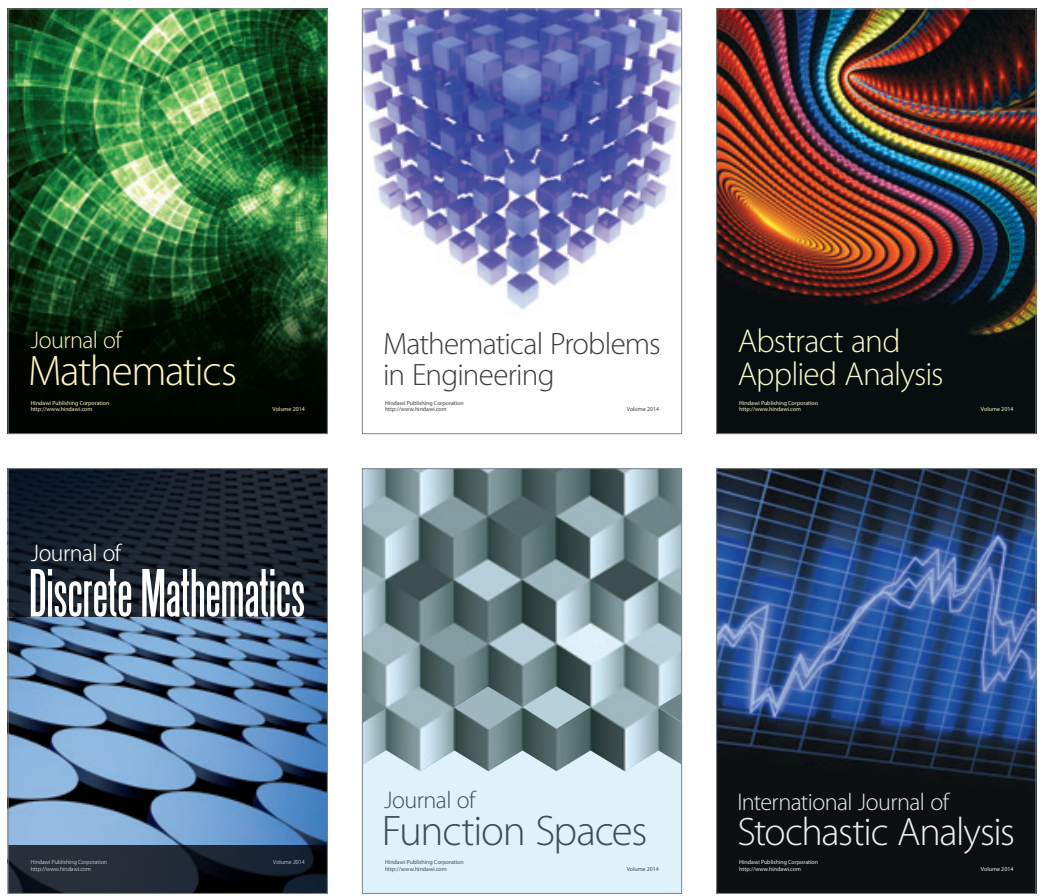

Journal of

Function Spaces

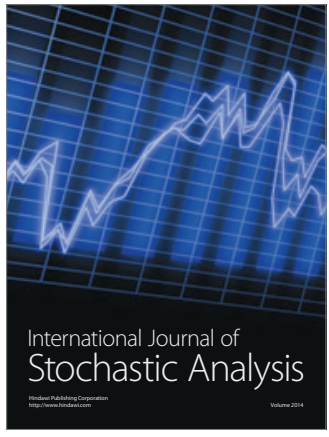

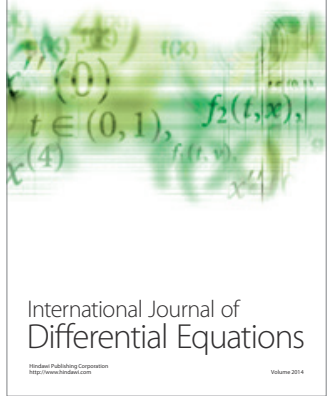
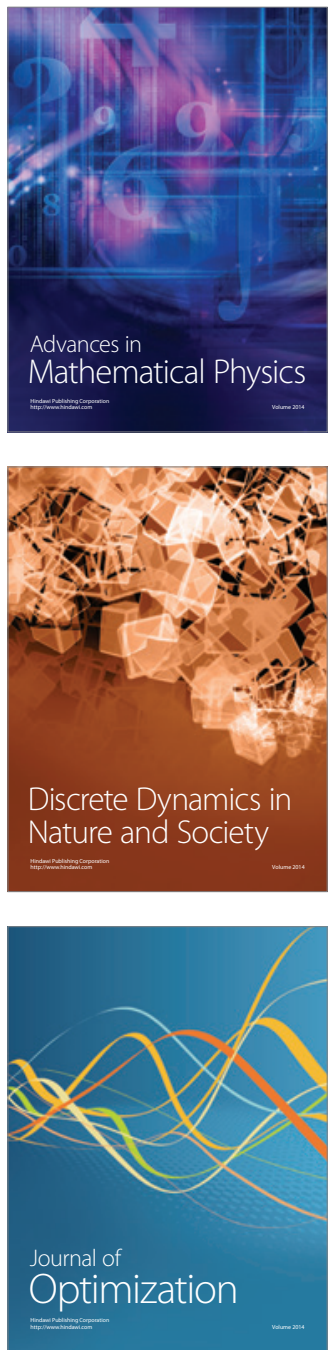